\title{
Optomechanical transport of cold atoms induced by structured light
}

\author{
Giuseppe Baio, ${ }^{*}$ Gordon R. M. Robb $\odot$, Alison M. Yao $\odot$, and Gian-Luca Oppo \\ SUPA and Department of Physics, University of Strathclyde, Glasgow G4 ONG, Scotland, United Kingdom
}

(Received 9 December 2019; accepted 24 March 2020; published 4 May 2020)

\begin{abstract}
Optomechanical pattern forming instabilities in a cloud of cold atoms lead to self-organized spatial structures of light and atoms. Here, we consider the optomechanical self-structuring of a cold atomic cloud in the presence of a phase structured input field, carrying orbital angular momentum. For a planar ring cavity setup, a model of coupled cavity field and atomic density equations describes a wide range of drifting modulation instabilities in the transverse plane. This leads to the formation of rotating self-organized rings of light-atom lattices. Using linear stability analysis and numerical simulations of the coupled atomic and optical dynamics, we demonstrate the presence of macroscopic atomic transport corresponding to the pattern rotation, induced by the structured pump phase profile.
\end{abstract}

DOI: 10.1103/PhysRevResearch.2.023126

\section{INTRODUCTION}

The spontaneous emergence of spatiotemporal order is a prominent feature of physical systems driven far from equilibrium [1]. Optical systems provide valuable platforms for studying spatial self-organization, where the self-sustained patterns are typically encoded in the internal excitations of a nonlinear medium [2,3]. Besides their fundamental interest, optical spatial structures offer potential applications in information processing such as optical memories and registers [4].

Recent experiments have provided paradigmatic examples of spatial self-organization in transverse nonlinear optics with cold atoms such as density, electronic, and magnetic spatial ordering [5-7]. In the first case, the bunching of atoms due to optomechanical forces can provide positive feedback and lead to spatial instabilities [8]. Other mechanisms for pattern formation in cold atoms rely instead on optical pumping and internal state nonlinearities. In the regime of quantum degeneracy, the nonequilibrium phase transition corresponding to the onset of spatial self-organization has been interpreted as a quantum phase transition $[9,10]$. Multimodal configurations have been shown to significantly enrich the physical scenario, allowing the realization of frustrated interactions and supersolid states with ultracold atomic gases [11,12].

The introduction of orbital angular momentum of light $(\mathrm{OAM})$ and its relative ease of generation and control paved the way to a plethora of applications in optical manipulation and information technologies $[13,14]$. Focusing on cold atoms, the use of OAM modes enables the transfer of angular momentum to both motional and internal atomic degrees of freedom [15]. Macroscopic rotation of cold atomic gases is

\footnotetext{
*giuseppe.baio@strath.ac.uk

Published by the American Physical Society under the terms of the Creative Commons Attribution 4.0 International license. Further distribution of this work must maintain attribution to the author(s) and the published article's title, journal citation, and DOI.
}

also achieved in the dispersive regime by means of rotating ring lattice trapping potentials $[16,17]$. Such geometries and related configurations, when applied to cold atoms, offer the possibility to engineer classical or quantum transport phenomena and simulate topological condensed matter effects such as fractional quantum Hall physics [18,19].

In this work we extend previous studies of transverse optomechanical instabilities in cold atomic clouds, allowing the input field to have a spatially dependent phase structure and carrying nonzero OAM. Externally controlled phase gradients are known to induce drifting pattern dynamics in the transverse plane, as demonstrated with hot atomic vapors [20,21], and can be applied to control the motion of spatial dissipative solitons [22-24]. Moreover, drifting dynamics induced by OAM was observed for patterned states in a single mirror system with a photorefractive nonlinear crystal [25].

For a cold atomic gas placed within a ring cavity under the action of an input beam with OAM and with a purely optomechanical nonlinearity, we show that the coupled lightatom dynamics in the plane transverse to the cavity axis results in circular drift motions of bunched atoms. This novel mechanism is inherently associated with the spontaneous breaking of a continuous rotational symmetry via pattern formation coupled to the optomechanics due to the input OAM and can be used to engineer transport of cold atoms via self-organized potentials with ring geometries for a wide range of parameters that are experimentally achievable. Our atomic ensemble is assumed to be kept at constant temperature by means of optical molasses, providing strong damping to the center-of-mass dynamics. Furthermore, dealing with density redistribution-induced interactions, atoms behave as a collection of linear scatterers. This opens the possibility of extending the present results to soft matter candidates for optomechanical self-organization such as suspensions of colloidal particles [26-28].

The paper is organized as follows. In Sec. II we review the known features of optomechanical self-structuring in a ring cavity for a spatially homogeneous input pump. In Sec. III we discuss drifting pattern dynamics induced by OAM in the 


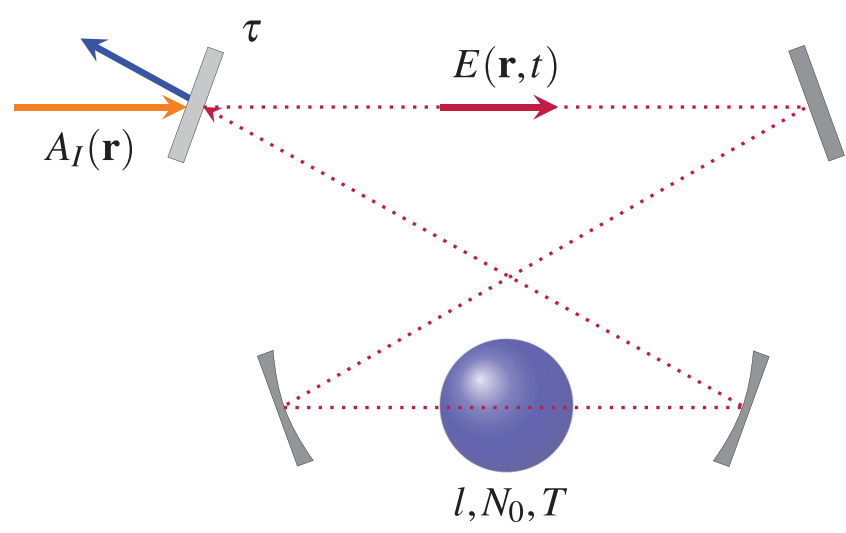

FIG. 1. Sketch of our cavity setup. A structured beam with amplitude $A_{I}(\mathbf{r})$ and phase $\exp (i l \varphi)$ drives a single longitudinal cavity mode. The ring cavity has effective length $L$ and mirror transmittivity $\tau$. The intracavity field $E(\mathbf{r}, t)$ interacts with a cloud of two-level atoms of thickness $l$, average atomic density $N_{0}$, and temperature $T$.

pump profile, which leads to the formation of self-organized rotating ring lattices. Finally, in Sec. IV, we compare our predictions with numerical results from particle dynamics simulations, showing the presence of macroscopic optomechanical transport corresponding to the pattern rotation.

\section{THE MODEL}

In this section we discuss a theoretical model, adapted from Refs. [29,30], describing the transverse dynamics of the cavity field and the density of the atomic cloud. A linear stability analysis provides the threshold condition for optomechanical modulation instabilities (MI) of the flat stationary states.

\section{A. Model equations}

We consider a cold thermal cloud of two-level atoms within a planar ring cavity geometry of effective length $L$, sketched in Fig. 1.

Theoretical models describing the coupled dynamics of cavity field and density modulations of a cold atomic gas involve the paraxial wave equation for a single longitudinal mode of the cavity field $E(\mathbf{r}, t)$ coupled to the atomic density distribution $N(\mathbf{r}, t)=N_{0} n(\mathbf{r}, t)$, where $N_{0}$ is the average density of the sample and $\mathbf{r}$ denotes the transverse spatial coordinate [30]. The field equation is derived in the slowly varying envelope, rotating wave, and mean-field approximations as follows [31]:

$$
\frac{\partial E}{\partial t}=-\kappa\left\{(1+i \theta) E+A_{I}(\mathbf{r})-\frac{\tilde{\gamma}}{1+|E|^{2}} n E+i a \nabla_{\perp}^{2} E\right\},
$$

where $\theta$ is the cavity-pump detuning, describing the linear shift on the cavity field, $A_{I}(\mathbf{r})$ represents a spatially dependent pump, and diffraction is described by the transverse Laplacian $\nabla_{\perp}^{2}$. Here we focus on a pump profile of the form $A_{I}(\mathbf{r})=$ $A_{I}(r) \exp (i l \varphi)$, where $A_{I}(r)$ is a generic radial profile and $r, \varphi$ are the radial and azimuthal coordinates, respectively. In addition, $\kappa=c \tau / L$ represents the cavity linewidth and $\sqrt{a}=\sqrt{\lambda L / 4 \pi \tau}$ the diffraction length, where $\lambda$ is the light wavelength, $\tau$ the mirror trasmissivity, and $c$ the speed of light in vacuum. The response of the two-level saturable medium ${ }^{1}$ is parametrized in terms of the complex susceptibility $\tilde{\gamma}=$ $2 C(1+i \Delta)$, with $\Delta=2 \delta / \Gamma$, where $\delta$ is the light detuning with respect to the atomic resonance. Finally, the parameter $C=b_{0} / 2 \tau\left(1+\Delta^{2}\right)$ is known as the cooperativity parameter and it depends on the optical thickness of the sample at resonance $b_{0}, \tau$, and $\Delta$. For our description of atomic motion, we focus on the limit when the population of the excited atomic state is negligible. This is captured by the saturation parameter $^{2} s=|E(\mathbf{r}, t)|^{2}$. In general, the resulting dipole force $\mathbf{f}_{\mathrm{dip}}$ acting on the atom center of mass can be derived from the AC Stark potential:

$$
\mathbf{f}_{\mathrm{dip}}=-\nabla_{\perp} U_{\mathrm{dip}}(\mathbf{r}, t)=-\frac{\hbar \Gamma \Delta}{4} \frac{\nabla_{\perp} s(\mathbf{r}, t)}{1+s(\mathbf{r}, t)} .
$$

Note the dependence of Eq. (2) on the detuning $\Delta$, such that the atom is trapped in high- (low-) intensity regions when $\Delta<0(\Delta>0)$. Assuming the presence of optical molasses, we adopt a classical treatment of positions $\mathbf{r}_{j}$ and momenta $\mathbf{p}_{j}(i, j=1, \ldots, N)$, leading to the canonical set of stochastic differential equations:

$$
\frac{d \mathbf{r}_{j}}{d t}=\frac{\mathbf{p}_{j}}{m}, \quad \frac{d \mathbf{p}_{j}}{d t}=\mathbf{f}_{\mathrm{dip}}\left(\mathbf{r}_{j}, t\right)-\gamma \frac{\mathbf{p}_{j}}{m}+\xi_{j}(t),
$$

where $m$ is the atomic mass, $\gamma$ describes friction (momentum damping), and $\xi_{j}(t)$ is a stochastic variable such that $\left\langle\xi_{j}(t)\right\rangle=0$ and $\left\langle\xi_{j}(t) \xi_{j}^{\prime}\left(t^{\prime}\right)\right\rangle=2 D_{p} \delta_{j j^{\prime}} \delta\left(t-t^{\prime}\right)$, with $D_{p}$ being the diffusion constant in the space of momenta. In the thermodynamic limit $(N \rightarrow \infty)$, the above equations map to the Chandrasekhar equation for the phase space distribution function $f(\mathbf{r}, \mathbf{p}, t)$ in the one-particle picture [27,32]:

$$
\frac{\partial}{\partial t} f=\frac{\mathbf{p}}{m} \cdot \nabla_{\perp} f+\mathbf{f}_{\mathrm{dip}}(\mathbf{r}, t) \cdot \nabla_{\mathbf{p}} f+\gamma\left[\nabla_{\mathbf{p}}(f \mathbf{p})+\frac{m}{\beta} \nabla_{\mathbf{p}}^{2} f\right],
$$

where $\beta=1 / k_{B} T$. Note that, in the presence of momentum damping, the collisional contribution in Eq. (4) is replaced by the term $\nabla_{\mathbf{p}}(f \mathbf{p})+\frac{m}{\beta} \nabla_{\mathbf{p}}^{2} f$, describing relaxation in momentum space. In the limit of strong viscous damping (more generally, when $t \gg 1 / \gamma$ ), the momentum $\mathbf{p}$ is eliminated from the dynamics and one derives the Smoluchowski equation for the density distribution $n(\mathbf{r}, t)$, by direct integration of Eq. (4) in momentum space, namely,

$$
\frac{\partial n}{\partial t}=-\beta D \nabla_{\perp} \cdot\left[n \mathbf{f}_{\mathrm{dip}}\right]+D \nabla_{\perp}^{2} n,
$$

where diffusive dynamics, as an effect of the optical molasses, is characterized by the constant $D=1 / \gamma m \beta$ [33]. Clearly, in the case of hot atoms $(\beta \rightarrow 0)$, the diffusive term dominates and one has $n(\mathbf{r}, t) \approx 1$, i.e., the homogeneous state.

\footnotetext{
${ }^{1}$ The characteristic dependence is obtained by adiabatic elimination of the atomic variables.

${ }^{2}$ The cavity field $E$ is rescaled by the saturation intensity at detuning $\Delta$, i.e., $\left[I_{\text {sat }}\left(1+\Delta^{2}\right)\right]^{1 / 2}$, where $I_{\text {sat }}$ represents the saturation intensity at resonance.
} 
Generally speaking, MI in our system is due to a competition between two-level and density-driven nonlinearities. In what follows, we perform a linear stability analysis, showing that density inhomogeneities alone are sufficient to achieve (optomechanical) MI. The features of the resulting self-organized states in the presence of OAM are discussed in Sec. III.

\section{B. Linear stability analysis}

The family of homogeneous stationary states of our system is simply found imposing that all derivatives in both Eqs. (1) and (5) vanish. For the cavity field, i.e., $\partial E_{0} / \partial t=0$ and $\nabla_{\perp}^{2} E_{0}=0$ in Eq. (1), this yields

$$
A_{I}=\left[1+i \theta+\frac{\tilde{\gamma}}{1+\left|E_{0}\right|^{2}}\right] E_{0}
$$

with $n_{0}=1$. A consequence of the two-level nonlinear term in Eq. (6) is that the homogeneous field intensity $\left|E_{0}\right|^{2}$, as a function of the pump intensity $A_{I}$, is not necessarily single valued [31]. It is easy to see that, in the low saturation limit, our model Eqs. (1) and (5) read as follows:

$$
\begin{gathered}
\frac{\partial E}{\partial t^{\prime}}=-(1+i \theta) E+A_{I}\left(\mathbf{r}^{\prime}\right)-2 i C \Delta n E+i \nabla_{\perp}^{2} E, \\
\frac{\partial n}{\partial t^{\prime}}=\sigma D^{\prime} \nabla_{\perp} \cdot\left[n \nabla_{\perp}|E|^{2}\right]+D^{\prime} \nabla_{\perp}^{2} n,
\end{gathered}
$$

where time and space coordinates are rescaled as $t^{\prime}=\kappa t, \mathbf{r}^{\prime}=$ $\mathbf{r} / \sqrt{a}, D^{\prime}=D / \kappa a$, while the constant $\sigma=\hbar \Gamma \Delta / 4 k_{B} T$ denotes an optomechanical coupling strength. Moreover, Eq. (6) now simply reads $A_{I}=[1+i(\theta+2 C \Delta)] E_{0}$, so that there is always a one-to-one correspondence between the values of $\left|A_{I}\right|^{2}$ and $\left|E_{0}\right|^{2}$ in the low saturation limit. Perturbations around steady-state values $E_{0}$ and $n_{0}$ are introduced in Fourier space as $\delta E\left(\mathbf{q}, t^{\prime}\right)=a e^{i\left(\mathbf{q} \cdot \mathbf{r}^{\prime}+v t^{\prime}\right)}+b^{*} e^{-i\left(\mathbf{q} \cdot \mathbf{r}^{\prime}+v^{*} t^{\prime}\right)}, \delta n\left(\mathbf{q}, t^{\prime}\right)=$ $c e^{i\left(\mathbf{q} \cdot \mathbf{r}^{\prime}+v t^{\prime}\right)}+c^{*} e^{-i\left(\mathbf{q} \cdot \mathbf{r}^{\prime}+v^{*} t^{\prime}\right)}$. Thus, defining the variable $\mathbf{x}=$ $(a, b, c)^{T}$, the linearized system obtained from Eqs. (7) and (8) can be cast in the usual matrix form $(\mathbf{M}-v \mathbb{I}) \mathbf{x}=\mathbf{0}$, where $\mathbf{M}$ is the following matrix:

$$
\mathbf{M}=i^{-1}\left(\begin{array}{ccc}
-1-i\left(\mathbf{q}^{2}+2 C \Delta+\theta\right) & 0 & -2 i C \Delta E_{0} \\
0 & -1+i\left(\mathbf{q}^{2}+2 C \Delta+\theta\right) & 2 i C \Delta E_{0}^{*} \\
-D^{\prime} \mathbf{q}^{2} \sigma E_{0} * & -D^{\prime} \mathbf{q}^{2} \sigma E_{0} & -D^{\prime} \mathbf{q}^{2}
\end{array}\right)
$$

By imposing the marginal stability condition, i.e., $v=0$ or $\operatorname{det}(\mathbf{M})=0$, one finds the following analytical threshold:

$$
\left|E_{0}\right|^{2}=\frac{1+\left(\mathbf{q}^{2}+\theta+2 C \Delta\right)^{2}}{4 C \Delta \sigma\left(\mathbf{q}^{2}+\theta+2 C \Delta\right)}
$$

leading to the critical wave vector $\mathbf{q}_{c}^{2}=1-(\theta+2 C \Delta)$. Here we study the linear stability properties by spanning two different parameter regions. The two instability diagrams in Fig. 2 show the dependence of the linear growth rate (GR) as a function of $\left|E_{0}\right|^{2}$ vs detuning $\Delta[\text { Fig. 2(a) }]^{3}$ and $\left|E_{0}\right|^{2}$ vs. temperature $T$ [Fig. 2(b)]. The values of the growth rate are obtained by computing numerically the spectrum of eigenvalues and eigenvectors of the matrix $\mathbf{M}$ in Eq. (9) for the critical wave vector $\mathbf{q}_{c} \cdot{ }^{4}$ Finally, the homogeneously pumped system spontaneously selects a hexagonal patterned state for values of $\left|E_{0}\right|$ close to the threshold in Eq. (10) [34]. We remark here that, assuming low saturation, purely optomechanical structures occur in the proximity of the threshold only since, for stronger pumping, the electronic nonlinearity becomes relevant.

\section{PATTERN DYNAMICS WITH STRUCTURED PHASE}

In this section we analyze the effect of the spatially dependent pump $A_{I}\left(\mathbf{r}^{\prime}\right)$ on the transverse geometry and dynamics of

\footnotetext{
${ }^{3}$ Only the case $\Delta>0$ is shown as we have symmetric behavior for $\Delta<0$.

${ }^{4}$ In our representation $\delta E\left(\mathbf{q}, t^{\prime}\right)=a e^{i\left(\mathbf{q} \cdot \mathbf{r}^{\prime}+v t^{\prime}\right)}+b^{*} e^{-i\left(\mathbf{q} \cdot \mathbf{r}^{\prime}+v^{*} t^{\prime}\right)}$, the modes with the fastest growth in time are those with the lowest values of $-\operatorname{Im}[v]$. Figure 2 shows density plots of $-\operatorname{Im}[v]$ as a function of field intensity and detuning (a) and temperature (b).
}

the patterned states of our system. Before presenting numerical results, we show how drifting pattern dynamics arises from Eqs. (7) and (8), based on the argument from Refs. [22,35].

\section{A. Drifting pattern dynamics}

In order to include OAM in our system, the pump rate $A_{I}\left(\mathbf{r}^{\prime}\right)$ must have an azimuthally dependent phase $\exp (i l \varphi)$, where $l \in \mathbb{Z}$. Indeed, the factor $\exp (i l \varphi)$ generates $l$ intertwined phase fronts and a nontrivial topological structure due to the phase singularity at $\mathbf{r}^{\prime}=0$ [14]. In general, any phase profile including the factor $\exp (i l \varphi)$ is responsible for a nonvanishing contribution of OAM through the transverse plane. Let us further simplify the analysis recalling that the stationary solution of Eq. (8) is given by the Gibbs distribution [29]:

$n_{\mathrm{eq}}\left(\mathbf{r}^{\prime}\right)=\frac{\exp \left[-\beta U_{\mathrm{dip}}\left(\mathbf{r}^{\prime}\right)\right]}{\int_{V} \exp \left[-\beta U_{\mathrm{dip}}\left(\mathbf{r}^{\prime}\right)\right]}=\frac{\exp \left[-\sigma\left|E\left(\mathbf{r}^{\prime}\right)\right|^{2}\right]}{\int_{V} \exp \left[-\sigma\left|E\left(\mathbf{r}^{\prime}\right)\right|^{2}\right]}$.

The steady states of the coupled system above MI can in principle be obtained by integrating numerically Eq. (7), simply eliminating the density distribution. Thus, Eq. (11) acts back as the nonlinear term in the field equation, namely [30],

$$
\frac{\partial E}{\partial t^{\prime}}=-(1+i \theta) E+A_{I}\left(\mathbf{r}^{\prime}\right)-2 i C \Delta n_{\mathrm{eq}}\left(|E|^{2}\right) E+i \nabla_{\perp}^{2} E,
$$

where we emphasize here the dependence of $n_{\mathrm{eq}}\left(\mathbf{r}^{\prime}\right)$ on the field intensity. Therefore, the coupled system of Eqs. (7) and (8) reduces now to Eq. (12) only. Let us consider the local transformation $E\left(\mathbf{r}^{\prime}, t^{\prime}\right)=\tilde{E}\left(\mathbf{r}^{\prime}, t^{\prime}\right) \exp (i l \varphi)$. The 

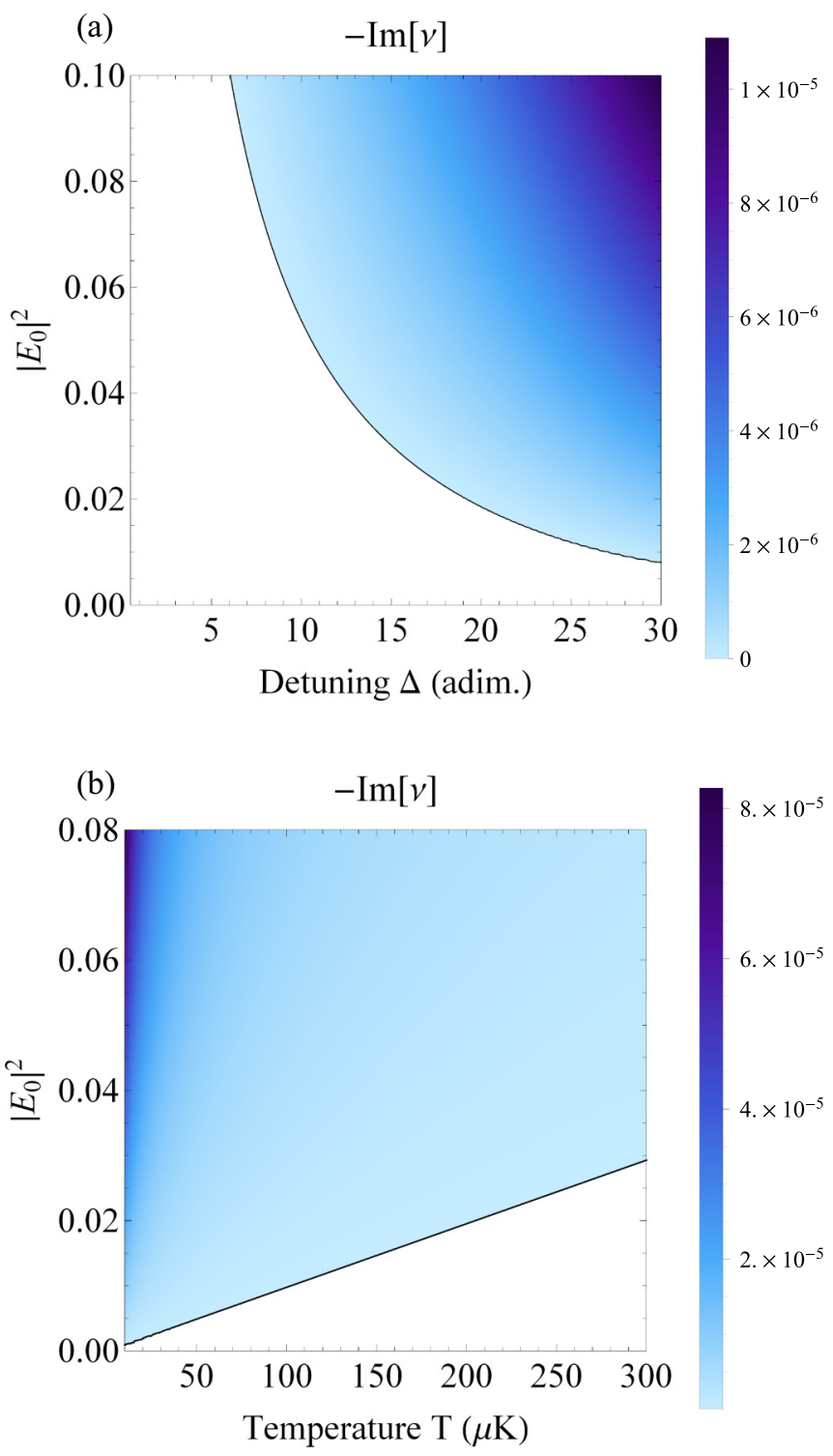

FIG. 2. 2D diagrams from linear stability analysis showing the growth rate $(-\operatorname{Im}[v])$ dependence in two parameter spaces. (a) GR as a function of $\left|E_{0}\right|^{2}$ and $\Delta$, sufficiently far from the atomic resonance (low saturation) at $\sigma=12$. (b) GR as a function of $\left|E_{0}\right|^{2}$ and temperature $T$, at $|\Delta|=20$. Parameters are chosen as follows: $\theta=-100, D^{\prime}=10^{-8}$, and $\mathbf{q}^{2}=\mathbf{q}_{c}^{2}$. Instability regions are obtained above the threshold in Eq. (10), represented here by the solid black line.

corresponding equation for $\tilde{E}\left(\mathbf{r}^{\prime}, t^{\prime}\right)$ reads now as follows:

$$
\begin{aligned}
\frac{\partial \tilde{E}}{\partial t^{\prime}}+\frac{2 l}{r^{\prime}} \nabla_{\perp} \tilde{E}= & -\left(1+\frac{l^{2}}{r^{\prime 2}}\right) \tilde{E}-i\left(\theta-\frac{l}{r^{\prime 2}}\right) \tilde{E} \\
& +A_{I}\left(r^{\prime}\right)-2 i C \Delta n_{\mathrm{eq}}\left(|\tilde{E}|^{2}\right) \tilde{E}+i \nabla_{\perp}^{2} \tilde{E} .
\end{aligned}
$$

One can recognize that the left-hand side of Eq. (13) has the form of a covariant derivative $D_{t^{\prime}}=\frac{\partial}{\partial t^{\prime}}+\mathbf{v}_{\mathrm{dr}} \cdot \nabla_{\perp}$, i.e., the expression of a time derivative in a locally comoving reference frame, according to the velocity $\mathbf{v}_{\mathrm{dr}}\left(\mathbf{r}^{\prime}\right)=2 l \nabla_{\perp} \varphi=$ $\frac{2 l}{r^{\prime}} \hat{\varphi}$. Thus, in the presence of OAM, the family of patterned solutions is seen in the laboratory frame drifting with a velocity $\mathbf{v}_{\mathrm{dr}}\left(\mathbf{r}^{\prime}\right)$ [22]. Furthermore, such an argument carries implications also for the geometry of the patterned state in our case. Indeed, the right-hand side of Eq. (13) shows a spatially dependent contribution to the decay rate and cavity detuning. Therefore, the spatial rigidity of the patterned phases is broken according to the spatial symmetry of the pump $A_{I}\left(\mathbf{r}^{\prime}\right)$ and the stationary solution is retrieved in a rotating reference frame defined (in polar coordinates) by $\tilde{\varphi}\left(r^{\prime}, t^{\prime}\right)=\varphi-\omega\left(r^{\prime}\right) t^{\prime}$, with the angular frequency $\omega\left(r^{\prime}\right)=2 l / r^{\prime 2}$. This corresponds to a differential rotation of the concentric patterned solutions at a fixed radius, as found in Ref. [35]. In what follows, we support the above observation by integrating Eq. (12) numerically.

\section{B. Numerical results}

We shall restrict here our analysis to a two-dimensional (2D) radial "top hat" profile with rapidly vanishing tails. A commonly used example is given by the following:

$$
A_{I}\left(\mathbf{r}^{\prime}\right)=\frac{A_{I}}{2}\left\{1-\tanh \left[\eta\left(r^{\prime}-r_{0}^{\prime}\right)\right]\right\} \exp (i l \varphi),
$$

where the constant $\eta>0$ determines the side steepness of the flat part with radius $r_{0}^{\prime}$. The advantage of using Eq. (14) is twofold: the used boundary conditions do not affect numerical results and allow us to investigate the transverse $2 \mathrm{D}$ dynamics induced by the structured phase factor $\exp (i l \varphi)$ on a wide area inside the integration domain. We integrate Eq. (12) using a Fourier split-step method. A domain of ten critical wavelengths is discretized in $256 \times 256$ points and time step $d t^{\prime}=5 \times 10^{-3}$. Figure 3 shows an example of optomechanical self-structuring with OAM index $l=1$. The presence of a helical phase $\exp (i l \varphi)$ in the pump profile (14) causes the field to vanish at $\mathbf{r}^{\prime}=0$ and induces the formation of diffractive rings whose intensity exceeds the MI threshold. By measuring peak distances in the radial profiles of the intensity [see Fig. 3(a)], we found that the diffractive rings are approximately spaced by the critical wavelength of the transverse MI although this is slightly perturbed by the radial size of the input pump. Since the intensity between contiguous rings is below the MI threshold, each ring undergoes a onedimensional (1D) angular instability independently, leading to a set of concentric ring lattice structures [35]. By varying $\eta$ and $r_{0}^{\prime}$, one can also influence the features of the patterned states. For example, increasing the steepness with $\eta$ may lead to outer diffraction rings faster achieving azimuthal MI. For any balanced choice of such parameters, self-structuring dynamics always leads to a set of ring light-density lattices, each one rotating independently from the others. Similar behavior is found with higher-order OAM modes $(l>1)$.

The self-organized light-atom ring lattices display differential rotation, in accordance to the angular frequency $\omega\left(r^{\prime}\right)=$ $2 l / r^{\prime 2}$. To show this, we consider the field in Eq. (12) at a fixed radius $r^{\prime}=r_{0}^{\prime}$, mapping the variation of $\varphi \in[0,2 \pi]$ to an effective $1 \mathrm{D}$ model with periodic boundary conditions and an input pump of the form $A_{I}(\varphi)=A_{I} e^{i l \varphi}$. The same argument is repeated in Sec. IV for studying atomic transport. Figure 4 shows a comparison, for $l=1,2,3$, of the rotation frequency estimated at several radial distances and showing good agreement with the predicted $\omega\left(r^{\prime}\right)$. Analogous 
(a)

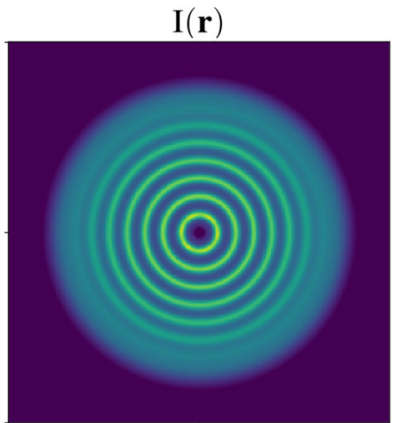

(b)

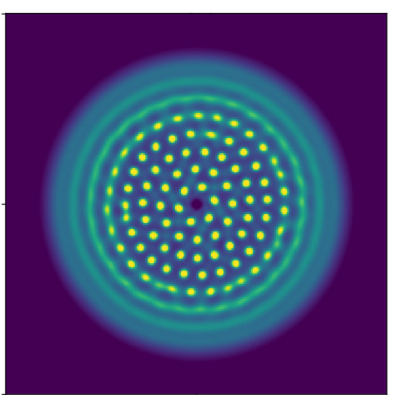

(c)
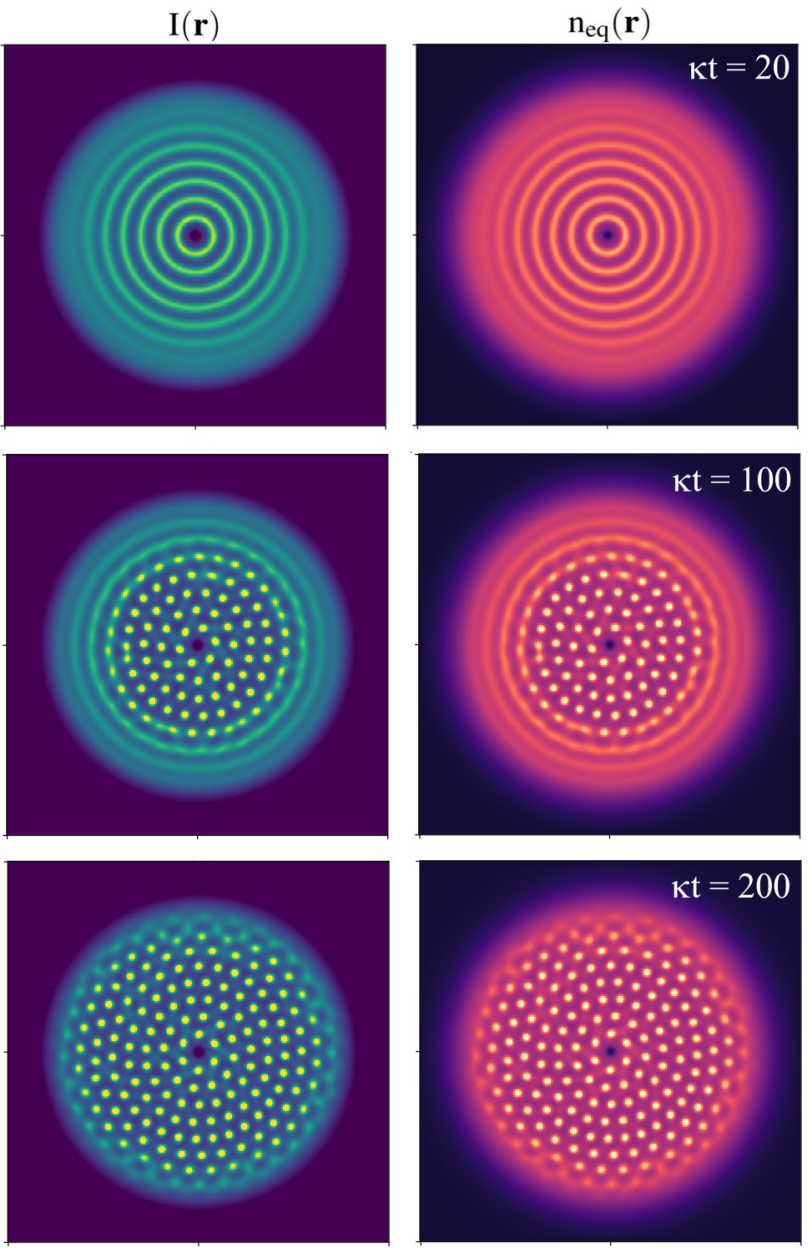

FIG. 3. Formation of bright self-organized ring lattices with a red detuned pump intensity $5 \%$ above threshold and with OAM $(l=1)$. The $2 \mathrm{D}$ intensity $I(\mathbf{r})$ (left) and density $n_{\mathrm{eq}}(\mathbf{r})$ (right) are shown at different times, i.e., $\kappa t=20(\mathrm{a}), \kappa t=100$ (b), and $\kappa t=200$ (c). Parameters are chosen as follows: $\theta=4, C \Delta=-3.5(\Delta<$ $0), \sigma=-25$.

rotational behavior can be expected for the case of bistable optomechanical dissipative solitons, as those found in [36]. OAM or other structured phase pump profiles can thus be used in this case to control the motion and the effective interactions of such localized dissipative structures $[23,24]$.

In the rest of this section, we discuss the effect of varying atomic diffusion on the ring lattice rotational dynamics. In order to take this dependence into account and obtain values of the rotation frequency comparable with experiments, we numerically integrate the coupled cavity equation (7) and the Smoluchowski equation (8), for the 1D azimuthal case at fixed radius. ${ }^{5}$ Note that, since we have $D=1 / \gamma m \beta$, any variation of $D$ at constant temperature is inversely proportional to a variation in the momentum damping coefficient $\gamma$, in the particle Eqs. (3). Results are shown in Fig. 5, where values of the estimated angular frequency are plotted against the

\footnotetext{
${ }^{5}$ By means of a second-order Crank-Nicolson scheme for coupled equations.
}

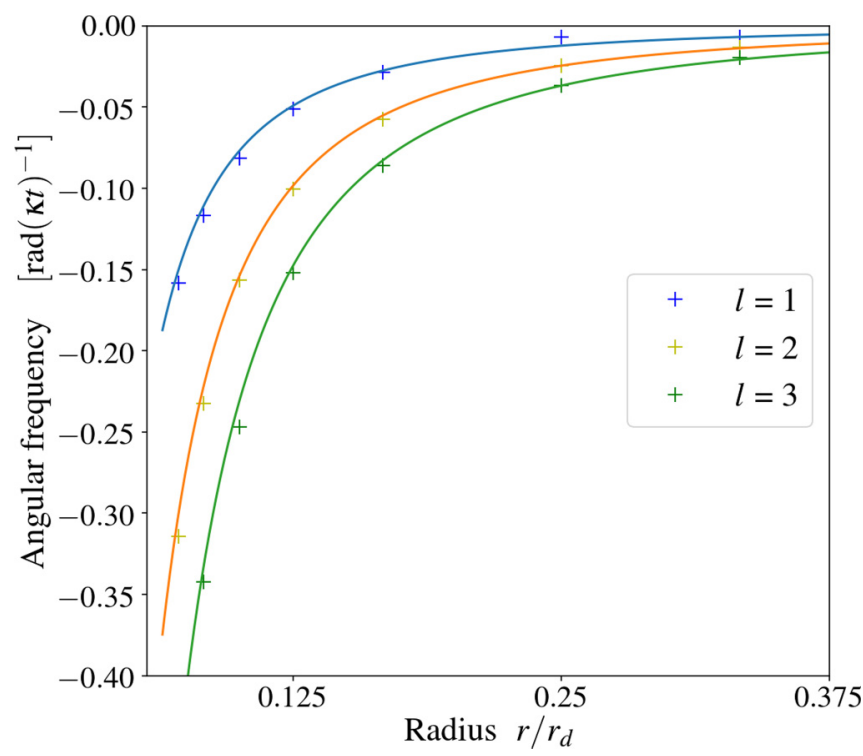

FIG. 4. Results from the 1D integration of Eq. (12). Values of the angular velocity of the patterned states are estimated at different radii (in units of half the domain size $r_{d}$ ). Each radius is chosen by simply adjusting the size of the integration domain. Solid lines represent the predicted angular frequencies $\omega$ for the three OAM cases $l=1,2,3$.

rescaled diffusion coefficient $D^{\prime}=D / \kappa a$. For higher values of $D^{\prime}$ (decreasing the friction $\gamma$ ), one observes a faster ring lattice rotation speed which saturates to a value roughly proportional to the index $l$. On the other hand, by increasing the friction $\gamma$, the rotation of the ring lattices created by the dipole interaction between atoms and light is affected by a drag, due to slower atomic diffusion. In the limit of very small diffusion

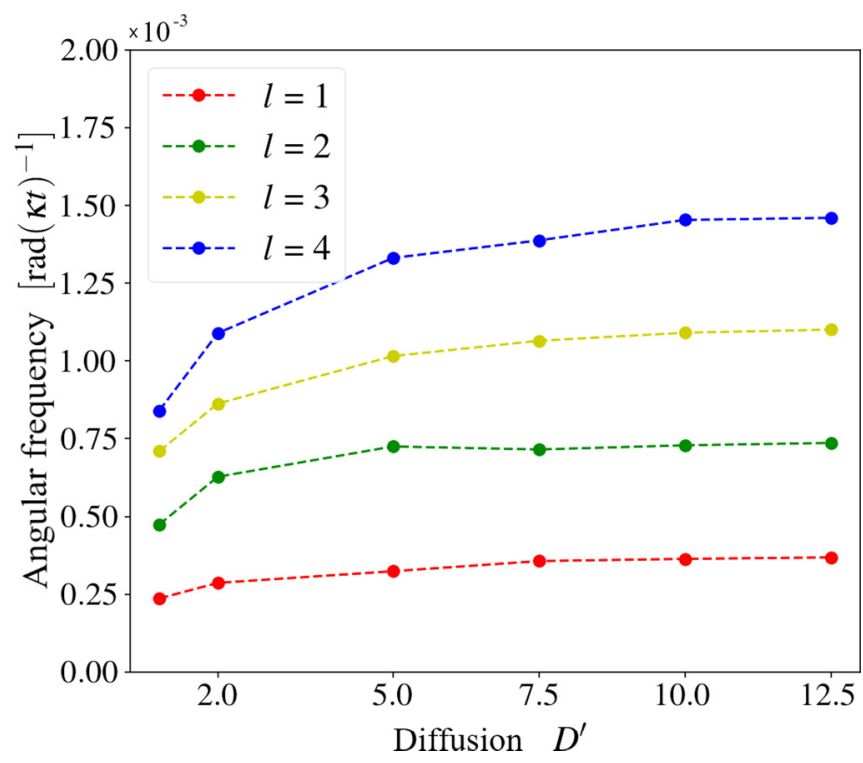

FIG. 5. Results from the 1D integration of the coupled model of Eqs. (7) and (8). Estimated values of the pattern rotation frequency are plotted against the rescaled diffusion coefficient $D^{\prime}$ (at fixed temperature $T$ ) for the cases $l=1, \ldots, 4$. As a result, faster diffusion processes imply higher values of experimentally measurable ring rotation frequency. 
$\left(D^{\prime} \ll 1\right)$, this drag can compensate the effect of the phase gradient introduced by the pump OAM and the concentric ring lattices would be expected to remain stationary. The above observations have profound consequences from the point of view of atomic center-of-mass dynamics since drifting selforganized light-density modulations induce transport of the self-trapped atoms, as shown in the next section.

\section{ATOMIC TRANSPORT}

In this section we demonstrate OAM-induced atomic transport corresponding to the rotation of the self-organized ring lattices. This is done by simply addressing the average momentum of the atomic distribution which quantifies the macroscopic rotational motion of the atoms, occurring at the onset of self-organization. We restrict ourselves to the 1D case, i.e., atoms confined in a ring geometry at a fixed radius within the cavity $2 \mathrm{D}$ transverse domain. Such a case can be realized experimentally by appropriately using Laguerre-Gauss beams of a given OAM in order to excite a single patterned ring [15].

\section{A. Average momentum}

We start our observations by recalling that, at equilibrium, the Chandrasekhar equation (4) for a dilute 1D atomic gas at constant temperature $T$ is solved by the Maxwell-Boltzmann distribution $\bar{f}(p)$, namely [37],

$$
\bar{f}(p)=\left(\frac{\beta}{2 \pi m}\right)^{3 / 2} \exp \left[-\frac{\beta(p-\langle p\rangle)^{2}}{2 m}\right],
$$

where $\langle p\rangle$ is the average (bulk) momentum around which the distribution $\bar{f}(p)$ is centered. Since we are interested in the strong friction limit, we assume that the total phase space distribution has the following factorized form [29,37]:

$$
f(r, p, t)=\bar{f}(p) n(r, t),
$$

where $r$ varies over a finite interval, depending on the chosen radius. Macroscopic transport in this regime can be addressed by looking at the average momentum $\langle p\rangle$, whose time evolution is obtained multiplying Eq. (4) by the momentum $p$ and integrating over momentum space [38]:

$$
\begin{aligned}
\frac{\partial n\langle p\rangle}{\partial t}= & \frac{1}{m} \int p^{2} \frac{\partial f}{\partial r} d p+\mathrm{f}_{\text {dip }} \int p \frac{\partial f}{\partial p} d p \\
& +\gamma \int p \frac{\partial}{\partial p}(p f) d p+\frac{\gamma m}{\beta} \int p \frac{\partial^{2} f}{\partial p^{2}} d p,
\end{aligned}
$$

where $f_{\text {dip }}$ is the dipole force in Eq. (2), expressed in the low saturation limit. We now rearrange Eq. (17), according to the following simple manipulations. Firstly, we have that $\int p^{2} \frac{\partial f}{\partial r} d p=\frac{\partial}{\partial r} n\left\langle p^{2}\right\rangle$, where $\left\langle p^{2}\right\rangle=(m \beta)^{-1}$ is the second moment of $\bar{f}(p)$ in Eq. (15). Moreover, $\int p \frac{\partial f}{\partial p} d p=-n$, $\int p \frac{\partial}{\partial p}(p f) d p=-n\langle p\rangle$, and $\int p \frac{\partial^{2} f}{\partial p^{2}} d p=0$, by simple calculus arguments. Thus, at equilibrium, Eq. (17) reads

$$
0=\frac{1}{m^{2} \beta} \frac{\partial}{\partial r} n(r, t)-\left[\mathrm{f}_{\mathrm{dip}}(r, t)+\gamma\langle p\rangle\right] n(r, t) .
$$

Atomic currents can thus be obtained integrating Eq. (18) along the 1D azimuthal domain. However, the first term in its right-hand side vanishes in our case ${ }^{6}$ so that, after integration, the average momentum finally reads

$$
\begin{aligned}
\langle p(t)\rangle_{\text {density }} & =-\frac{1}{\gamma m} \int \mathrm{f}_{\text {dip }}(r, t) n(r, t) d r \\
& =-D \beta \int \mathrm{f}_{\text {dip }}(r, t) n(r, t) d r .
\end{aligned}
$$

This quantifies the steady-state mass current along a rotating ring in the overdamped limit, from the knowledge of $n(r, t)$ only. In what follows, we compare $\langle p(t)\rangle_{\text {density }}$ with the ensemble averages obtained from simulating particle dynamics.

\section{B. Particle model}

Since a critical wave number $q_{c}$ is selected at the MI threshold of Eq. (7), it is convenient to redefine here atomic position $\bar{r}_{j}=q_{c} r_{j}$ and momenta $\bar{p}=p_{j} / \hbar q_{c}$, so that the set of particle equations (3) in 1D now reads [39]

$$
\begin{aligned}
& \frac{d \bar{r}_{j}}{d t^{\prime}}=2 \omega_{p} \bar{p}_{j}, \\
& \frac{d \bar{p}_{j}}{d t^{\prime}}=-\frac{\Gamma \Delta}{4 \kappa} \frac{\partial}{\partial \bar{r}}\left|E\left(\bar{r}=\bar{r}_{j}, t^{\prime}\right)\right|^{2}-\bar{\gamma} \bar{p}_{j}+\bar{\xi}_{j}\left(t^{\prime}\right),
\end{aligned}
$$

where $\omega_{p}=\hbar q_{c} / 2 m \kappa$ defines characteristic energy scales of an atom oscillating in a standing-wave potential of modulation $q_{c}$. In the overdamped case, one imposes momenta $\bar{p}_{j}$ at their steady-state values, leading to the following expression of the average momentum or atomic current:

$$
\left\langle\bar{p}_{j}\left(t^{\prime}\right)\right\rangle_{\text {particles }}=-\frac{\Gamma \Delta}{4 \kappa \bar{\gamma}}\left\langle\frac{\partial}{\partial \bar{r}}\left|E\left(\bar{r}=\bar{r}_{j}, t^{\prime}\right)\right|^{2}\right\rangle
$$

i.e., simply obtained as an ensemble average. Most importantly, this provides an expression of the relative constants in Eq. (5) in terms of microscopic parameters:

$$
D^{\prime}=\frac{4 \omega_{q}^{2}}{\bar{\gamma}^{2}} D_{p}^{\prime}=\frac{4 \omega_{q}^{2}}{\bar{\gamma}} \zeta_{p}^{2}, \quad \sigma=\frac{\omega_{q} \Gamma \Delta}{2 \bar{\gamma} D^{\prime}}=\frac{\Gamma \Delta}{8 \kappa \omega_{q}^{2} \zeta_{p}^{2}},
$$

where the diffusion $D^{\prime}$ and $\sigma$ have been expressed in terms of the (scaled) momentum spread $\zeta_{p}^{2}$. Note that $D_{p}^{\prime}=\bar{\gamma} \zeta_{p}^{2}$. We now integrate numerically the particle equations (20) in the overdamped limit. Those are coupled to the field equation in $1 \mathrm{D}$ through a density profile $n\left(\bar{r}, t^{\prime}\right)$, numerically reconstructed at all times from the particle positions $\bar{r}_{j}\left(t^{\prime}\right)$.

A typical outcome of our simulations is presented in Figs. 6(a) and 6(b), for the case of $5 \times 10^{4}$ atoms. Consistently with the 2D numerical integration in Sec. III, we observe drifting pattern dynamics in the 1D azimuthal geometry, i.e., ring lattice rotation at a fixed radius, induced by the OAM carried by the input pump. The results displayed in Fig. 7 show that, at the onset of self-organization, ${ }^{7}$ the atoms spontaneously develop net mass current along 1D rings, in the presence of OAM. This corresponds to the slope (drift) of the patterned field intensity $I\left(\bar{r}, t^{\prime}\right)$ and atomic density

\footnotetext{
${ }^{6}$ Since the density $n(r, t)$ is always a periodic function in our azimuthal domain at fixed radius.

${ }^{7}$ This happens at roughly $t^{\prime}=\kappa t \approx 5$ in our case.
} 

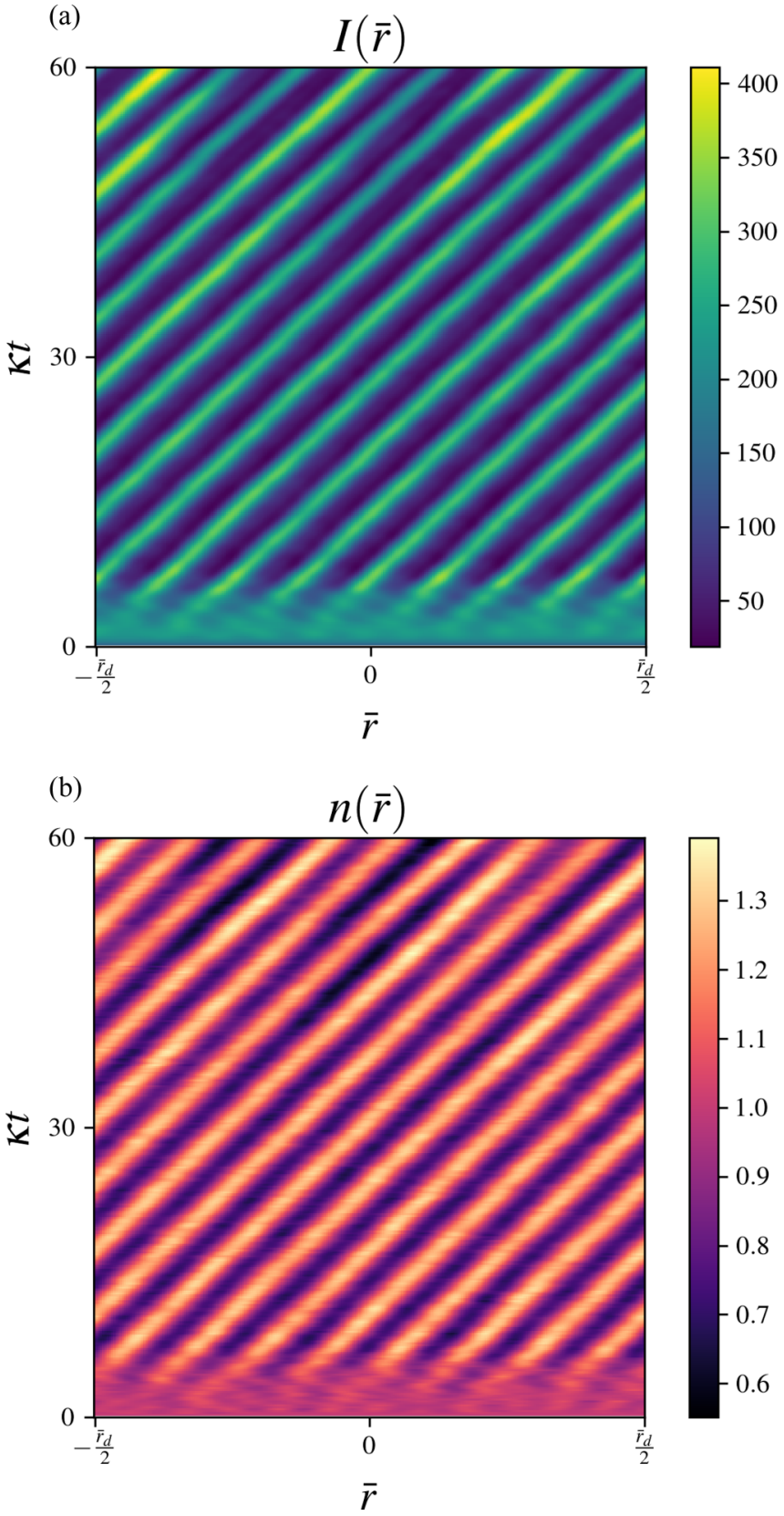

FIG. 6. Numerical results from the 1D particle dynamics simulation for a set of $5 \times 10^{4}$ atoms. (a) $\kappa t$ vs $\bar{r}$ plot showing the time evolution $\left(\kappa t_{\max }=60\right)$ of the 1D cavity field intensity $I(\bar{r})$. (b) Selfordering of the density $n(\bar{r}, t)$, numerically reconstructed from the particle trajectories. For blue detuned light $(\Delta>0)$, atoms bunch in the minima of $I(\bar{r})$. The OAM-dependent slope of the patterned phase in this graph corresponds to the rotation of the pattern in a ring geometry. Parameters are chosen as follows: OAM index $l=3, \frac{\Gamma}{\kappa}=$ $\bar{\omega}_{p}=\bar{\gamma}=1, \zeta_{p}=0.707, \Delta=100$, such that $D^{\prime} \approx 2, \sigma \approx 25$.

$n\left(\bar{r}, t^{\prime}\right)$, displayed in the $t^{\prime}$ vs $\bar{r}$ plots in Fig. 6. In particular, in Fig. 7(a), we compare the values of the current $\left\langle\bar{p}_{j}\left(t^{\prime}\right)\right\rangle$ (in units of $\hbar q_{c}$ ) obtained by means of both the above approaches, i.e., from Eq. (19) (density) and Eq. (21) (particles). In both cases $(l=3,4)$, one observes an exponential-like increase of $\left\langle\bar{p}_{j}\left(t^{\prime}\right)\right\rangle$, which eventually evolves to fluctuations around a steady-state value. Moreover, the two series show a highly

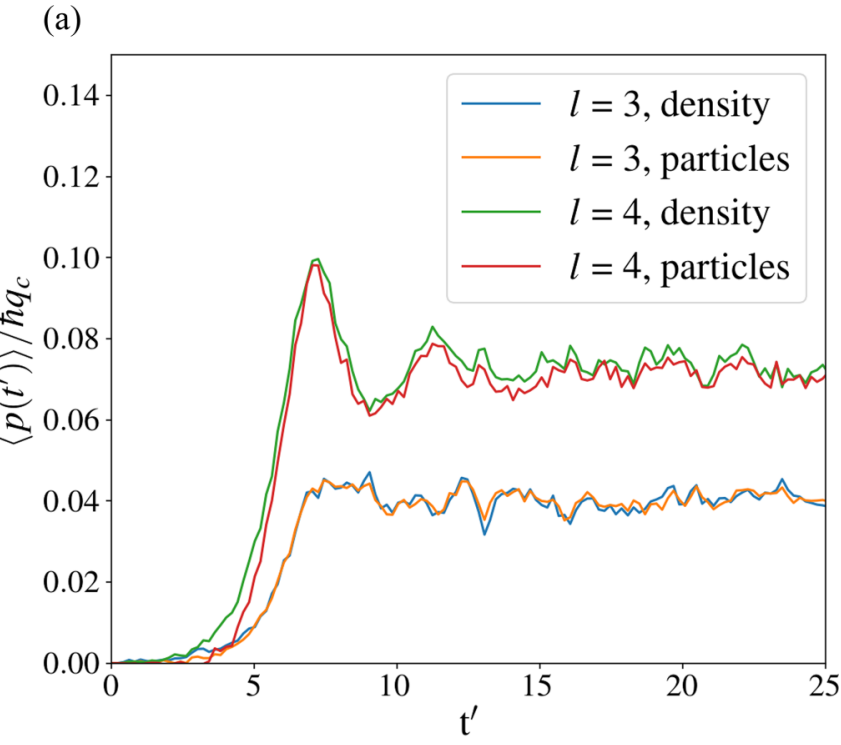

(b)

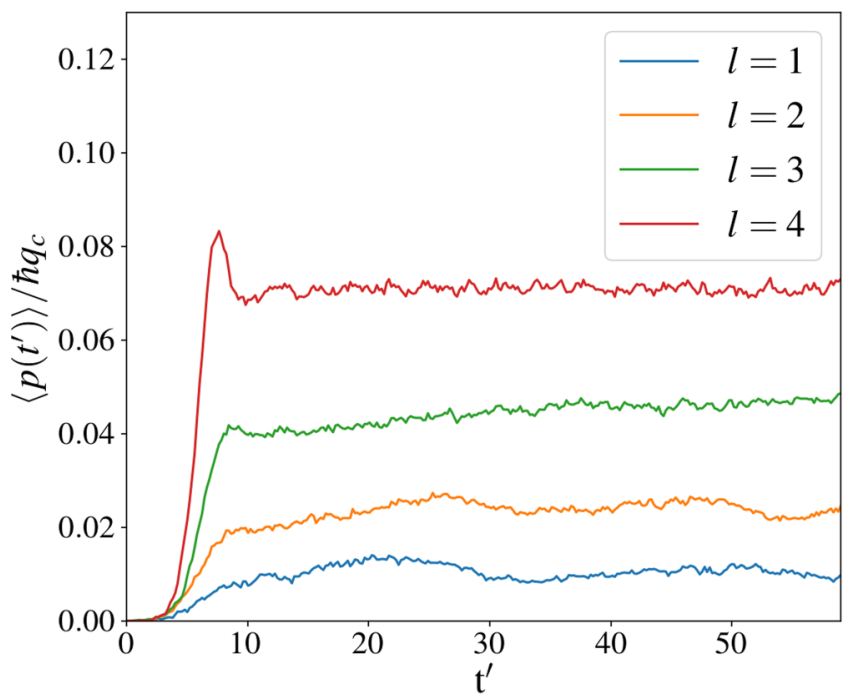

FIG. 7. Results from the 1D particle dynamics simulations. Atoms initially at rest and homogeneously distributed in the 1D domain display nonzero atomic current at the onset of self-organization. (a) Time evolution of the atomic current $\left\langle\bar{p}_{j}\left(t^{\prime}\right)\right\rangle$, in units of $\hbar q_{c}$, with OAM index $l=3,4$, comparing the values arising from the ensemble average in Eq. (19) and from the reconstructed density profile $n(\bar{r}, t)$ in Eq. (21). (b) Evolution to steady-state values of the atomic current, in units of $\hbar q_{c}$ at different values of the OAM index $l$. Each plotted line is in turn obtained by averaging over a set of ten identical launches for each $l$. These results demonstrate the presence of atomic transport along the 1D azimuthal rings, induced by the pump OAM.

correlated behavior. Finally, as shown in Fig. 7(b), in order to obtain more precise values of the steady-state currents $\left\langle\bar{p}_{j}\left(t^{\prime}\right)\right\rangle$ for different OAM indices $l=1, \ldots, 4$, we averaged over a set of ten simulations with the same set of parameters for each value of $l$. As a final remark, we observe from Fig. 7(b), that the steady-state current values increase nonlinearly with the OAM index $l$. This might suggest that the optomechanical transfer of OAM considered here, i.e., from the cavity field 
to the atoms, occurs more efficiently with higher-order OAM modes input. However, a detailed analysis of this mechanism in such a configuration is out of the scope of this work and will be investigated in future studies.

\section{CONCLUDING REMARKS}

Clouds of cold atoms in optical cavities, under the action of a coherent beam of light carrying OAM, can spontaneously form concentric rings of rotating light-atom lattices in the transverse plane, by means of optomechanical self-structuring (see Fig. 3). The rotation of these spatiotemporal structures is due to the gradient of the transverse phase distribution externally imposed on the pump beam. The observed angular velocity is directly proportional to the OAM and inversely proportional to the square of the ring radius (see Fig. 4). Moreover, for increasing friction and decreasing diffusion, the atoms introduce a drag that slows down the rotation of the patterned rings (see Fig. 5). By means of numerical evidences, we showed that such ring lattice rotation sustains macroscopic atomic motion along azimuthal $1 \mathrm{D}$ rings in the $2 \mathrm{D}$ transverse domain (see Figs. 6 and 7). Therefore, optomechanical transport and azimuthal mass currents induced by the OAM in the structured pump have been predicted for a cold thermal gas in the assumption of overdamped motion. A possible generalization of the case considered here is that of rotating optomechanical instabilities without momentum damping [40]. Similarly, extensions to the dynamics of optomechanical dissipative solitons and their interaction in the presence of an OAM carrying pump are also of interest for future theoretical studies and experimental realizations. Finally, our results can be applied to the case of a quantum degenerate gas, in order to study vortex formation and turbulent regimes [41]. The OAM transfer mechanism discussed here represents a potential platform to induce persistent currents of ultracold atoms, where such states are indeed an ideal candidate for the realization of atomtronic devices in circular atomic traps $[14,15]$.

\section{ACKNOWLEDGMENTS}

We are grateful to T. Ackemann and S. Franke-Arnold for helpful discussions. All authors acknowledge financial support from the European Training Network ColOpt, which is funded by the European Union (EU) Horizon 2020 program under the Marie Skłodowska-Curie Action, Grant Agreement No. 721465 .
[1] M. C. Cross and P. C. Hohenberg, Pattern formation outside of equilibrium, Rev. Mod. Phys. 65, 851 (1993).

[2] A. J. Scroggie, W. J. Firth, G. S. McDonald, M. Tlidi, R. Lefever, and L. A. Lugiato, Pattern formation in a passive Kerr cavity, Chaos, Solitons Fractals 4, 1323 (1994).

[3] F. T. Arecchi, S. Boccaletti, and P. Ramazza, Pattern formation and competition in nonlinear optics, Phys Rep. 318, 1 (1999).

[4] T. Ackemann, W. J. Firth, and G.-L. Oppo, Fundamentals and applications of spatial dissipative solitons in photonic devices, Adv. At., Mol., Opt. Phys. 57, 323 (2009).

[5] G. Labeyrie, E. Tesio, P. M. Gomes, G.-L. Oppo, W. J. Firth, G. R. M. Robb, A. S. Arnold, R. Kaiser, and T. Ackemann, Optomechanical self-structuring in a cold atomic gas, Nat. Photonics 8, 321 (2014).

[6] A. Camara, R. Kaiser, G. Labeyrie, W. J. Firth, G.-L. Oppo, G. R. M. Robb, A. S. Arnold, and T. Ackemann, Optical pattern formation with a two-level nonlinearity, Phys. Rev. A 92, 013820 (2015).

[7] G. Labeyrie, I. Krešić, G. R. M. Robb, G.-L. Oppo, R. Kaiser, and T. Ackemann, Magnetic phase diagram of lightmediated spin structuring in cold atoms, Optica 5, 1322 (2018).

[8] H. Ritsch, P. Domokos, F. Brennecke, and T. Esslinger, Cold atoms in cavity-generated dynamical optical potentials, Rev. Mod. Phys. 85, 553 (2013).

[9] K. Baumann, C. Guerlin, F. Brennecke, and T. Esslinger, Dicke quantum phase transition with a superfluid gas in an optical cavity, Nature (London) 464, 1301 (2010).

[10] G. R. M. Robb, E. Tesio, G.-L. Oppo, W. J. Firth, T. Ackemann, and R. Bonifacio, Quantum Threshold for Optomechanical Self-Structuring in a Bose-Einstein Condensate, Phys. Rev. Lett. 114, 173903 (2015).
[11] S. Gopalakrishnan, B. L. Lev, and P. M. Goldbart, Emergent crystallinity and frustration with Bose-Einstein condensates in multimode cavities, Nat. Phys. 5, 845 (2009).

[12] J. Léonard, A. Morales, P. Zupancic, T. Esslinger, and T. Donner, Supersolid formation in a quantum gas breaking a continuous translational symmetry, Nature (London) 543, 87 (2017).

[13] L. Allen, M. W. Beijersbergen, R. J. C. Spreeuw, and J. P. Woerdman, Orbital angular momentum of light and the transformation of Laguerre-Gaussian laser modes, Phys. Rev. A 45, 8185 (1992).

[14] A. M. Yao and M. J. Padgett, Orbital angular momentum: Origins, behavior and applications, Adv. Opt. Photonics 3, 161 (2011).

[15] S. Franke-Arnold, Optical angular momentum and atoms, Philos. Trans. R. Soc. A 375, 2087 (2017).

[16] S. Franke-Arnold, J. Leach, M. J. Padgett, V. E. Lembessis, D. Ellinas, A. J. Wright, J. M. Girkin, P. Öhberg, A. S. Arnold, Optical ferris wheel for ultracold atoms, Opt. Express 15, 8619 (2007).

[17] A. S. Arnold, Extending dark optical trapping geometries, Opt. Lett. 37, 2505 (2012).

[18] P. Hänggi and F. Marchesoni, Artificial Brownian motors: Controlling transport on the nanoscale, Rev. Mod. Phys. 81, 387 (2009).

[19] M. Łącki, H. Pichler, A. Sterdyniak, A. Lyras, V. E. Lembessis, O. Al-Dossary, J. C. Budich, and P. Zoller, Quantum Hall physics with cold atoms in cylindrical optical lattices, Phys. Rev. A 93, 013604 (2016).

[20] G. Grynberg, Drift instability and light-induced spin waves in an alkali vapor with feedback mirror, Opt. Commun. 109, 483 (1994). 
[21] J. P. Seipenbusch, T. Ackemann, B. Schäpers, B. Berge, and W. Lange, Drift instability and locking behavior of optical patterns, Phys. Rev. A 56, R4401 (1997).

[22] W. J. Firth and A. J. Scroggie, Optical Bullet Holes: Robust Controllable Localized States of a Nonlinear Cavity, Phys. Rev. Lett. 76, 1623 (1996).

[23] F. Pedaci, P. Genevet, S. Barland, M. Giudici, and J. Tredicce, Positioning cavity solitons with a phase mask, Appl. Phys. Lett. 89, 221111 (2006).

[24] C. Cleff, B. Gütlich, and C. Denz, Gradient Induced Motion Control of Drifting Solitary Structures in a Nonlinear Optical Single Feedback Experiment, Phys. Rev. Lett. 100, 233902 (2008).

[25] V. Caullet, N. Marsal, D. Wolfersberger, and M. Sciamanna, Vortex Induced Rotation Dynamics of Optical Patterns, Phys. Rev. Lett. 108, 263903 (2012).

[26] P. Smith, A. Ashkin, and W. J. Tomlinson, Four-wave mixing in an artificial Kerr medium, Opt. Lett 6, 284 (1981).

[27] D. Rogovin and S. O. Sari, Phase conjugation in liquid suspensions of microspheres in the diffusive limit, Phys. Rev. A 31, 2375 (1985).

[28] P. J. Reece, E. M. Wright, and K. Dholakia, Experimental Observation of Modulation Instability and Optical Spatial Soliton Arrays in Soft Condensed Matter, Phys. Rev. Lett. 98, 203902 (2007).

[29] M. Saffman and Y. Wang, Lect. Notes Phys. 751, 361 (2008).

[30] E. Tesio, G. R. M. Robb, T. Ackemann, W. J. Firth, and G.-L. Oppo, Spontaneous optomechanical pattern formation in cold atoms, Phys. Rev. A 86, 031801(R) (2012).

[31] L. A. Lugiato and C. Oldano, Stationary spatial patterns in passive optical systems: Two-level atoms, Phys. Rev. A 37, 3896 (1988)
[32] S. Chandrasekhar, Stochastic problems in physics and astronomy, Rev. Mod. Phys. 15, 1 (1943).

[33] T. W. Hodapp, C. Gerz, C. Furtlenher, C. I. Westbrook, W. D. Phillips, and J. Dalibard, Three-dimensional spatial diffusion in optical molasses, Appl. Phys. B 60, 135 (1995).

[34] W. J. Firth, A. J. Scroggie, G. S. McDonald, and L. A. Lugiato, Hexagonal patterns in optical bistability, Phys. Rev. A 46, R3609 (1992).

[35] A. M. Yao, C. J. Gibson, and G.-L. Oppo, Control of spatially rotating structures in diffractive Kerr cavities, Opt. Express 27, 31273 (2019)

[36] E. Tesio, G. R. M. Robb, T. Ackemann, W. Firth, and G.-L. Oppo, Dissipative solitons in the coupled dynamics of light and cold atoms, Opt. Express. 21, 26144 (2013).

[37] K. Huang, Statistical Mechanics, 2nd ed. (Wiley, New York, 1987).

[38] R. W. Davies, The Connection between the Smoluchowski Equation and the Kramers-Chandrasekhar Equation, Phys. Rev. 93, 1169 (1954).

[39] G. R. M. Robb, N. Piovella, A. Ferraro, R. Bonifacio, P. W. Courteille, and C. Zimmermann, Collective atomic recoil lasing including friction and diffusion effects, Phys. Rev. A 69, 041403(R) (2004).

[40] E. Tesio, G. R. M. Robb, T. Ackemann, W. J. Firth, and G.-L. Oppo, Kinetic Theory for Transverse Optomechanical Instabilities, Phys. Rev. Lett. 112, 043901 (2014).

[41] M. C. Tsatsos, P. E. S. Tavares, A. Cidrim, A. R. Fritsch, M. A. Caracanhas, F. E. A. dos Santos, C. F. Barenghi, and V. S. Bagnato, Quantum turbulence in trapped atomic Bose-Einstein condensates, Phys. Rep. 622, 1 (2016). 